\title{
Environmental Conditions and Performance Outcomes: A Preliminary Report of Implications for Patient Outcomes
}

Joseph D. Fortin, DO' ${ }^{1}$, Donna Holland, $\mathrm{PhD}^{2}$, and Erin Korreck, $\mathrm{MA}^{2}$

From: ${ }^{1}$ Spine Technology and Rehabilitation, Fort Wayne, IN, and Clinical Professor of Physical Medicine and Rehabilitation, Indiana University School of Medicine, Fort Wayne, Fort Wayne,IN.

Dr. Fortin is Medical Director of Spine

Technology and Rehabilitation, Fort Wayne, IN; and Clinical Professor, Indiana University School of Medicine. Dr. Holland is Assistant Professor, Sociology Department, Indiana University Purdue University Fort Wayne, Fort Wayne, IN. Ms. Korreck is graduate student at Indiana University/Purdue University, Fort Wayne, IN.

Address correspondence: Joseph D. Fortin, DO Medical Director Spine Technology and Rehabilitation 7230 Engle Road, Suite 210 Fort Wayne, IN 46804 E-mail: fortin@pol.net

Disclaimer: There was no external funding in the preparation of this manuscript. Conflict of interest: None.

Manuscript received: 05/15/2008 Revised manuscript received: 07/10/2008 Accepted for publication: $08 / 20 / 2008$

Free full manuscript: www.painphysicianjournal.com
Background: Whereas a host of studies have established various forms of experimental bias, few clinical investigations have examined the relationship of the behavior of the observer or examiner to a subject's physical performance.

Objective: To measure the grip strength of volunteers in 2 distinct clinical "environments."

Methodology: Twenty subjects were randomized in a crossover design to undergo grip strength testing in positive and negative environments as created by the distinctly different behavioral/communication approaches of the research staff. Each subject underwent 4 consecutive trials in both settings.

Results: A paired t- test was conducted to determine if the contrasting environments impacted the volunteer's performance. Eight of the 10 subjects demonstrated a significantly stronger grip in the positive environment. One subject's grip remained essentially unchanged and one subject provided a slightly higher performance in the negative setting.

Conclusions: The study suggests that clinical environmental conditions influence the physical performance of a grip strength maneuver. Thus, it seems probable that clinical or experimental settings may affect diagnostic test results and/or functional outcome.

Key words: environment, improved outcomes, support, physicians' attitudes

Pain Physician 2008; 11:5:655-658 eonard and Matsatu (1) demonstrated that when physicians were observed by research staff members quality of care ratings diminished after an average of 10 to 15 patient visits. The quality of care also decreased with the absence of the research staff. The study suggests that physician behavior can be affected by situational variables which consequently may alter quality of care.
Another study found patients were more satisfied with their care if they shared control with physicians as active participants in their care versus patients who allowed the doctor sole control (2). Moreover, sex $(3,4)$, physician age (5), attire, and communication style (6) are all factors that may impact patient satisfaction ratings. Clearly many non-medical variables affect outcome in a clinical setting and may influence measurements in clinical experimental environments. 


\section{Objective}

The present study was designed to determine if the behavioral approaches and communication styles of research staff could influence functional outcome of test subjects in contrasting experimental environments.

\section{Methodology}

Ten subjects were randomized in a crossover design to undergo grip strength testing in 2 settings one labeled the positive environment and the other designated as the negative environment. The subjects were not informed of the contrasting testing environments. The exclusion criteria included past medical history such as a cardiopulmonary disorder, complications of prior surgery, trauma, loss of strength or range of motion, and any factor that would preclude or hamper optimal performance during the testing protocol.

Grip strength was measured in 10 volunteers, ages ranging from 24 to 56 (Table 1), using a calibrated Jamar hand dynamometer (model $5030 \mathrm{KIT}$ ) and a standard technique (Ashton and Myers 2004) in 2 environments (7).

When the subjects entered the positive environment the examiner greeted the subjects warmly remarking, "Don't worry we're going to get you through this," and proceeded to read the subjects the testing instructions while maintaining positive eye contact. The participants' grip strength was then tested on the hand dynamometer for 4 trials. Conversely when the subjects entered the negative environment the administrator cast his gaze downward and asked, "Are you going to be able to do this?"

The examiner then proceeded to read the subjects the instructions and tested the grip strength with 4 trials, absent eye contact. The trials were separated by 15 second rest periods commensurate with the recommendations from a previous investigation (8).

\section{Results}

A paired T-test was conducted to determine whether the contrasting environments impacted the performance of the volunteers. The test scores for each subject in both environments are delineated in Table 1. A paired t-test disclosed a significant difference between the positive and negative environments at a .05 level suggesting that in the positive environment the subjects' grip strength was significantly higher than in the negative environment. Eight of the 10 subjects demonstrated consistently higher grip strength in the positive environment, with one subject providing a slightly higher performance in the negative setting.

\section{Discussion}

This study demonstrates that the subjects' grip strength was significantly stronger in the positive versus negative setting — suggesting that the clinical ambience or diagnostic testing environment may impact research results and functional outcome.

Although several investigators have demonstrated a potential link between the clinical research setting and physiological or behavioral changes - we were unable to find another study that related the testing environment to a physical performance variable with subjects in a clinical setting.

Outcome and quality assurance measures have become integral features of the highly regulated and competitive healthcare world. Likewise, bias and variability control are standard elements of today's research milieu. While numerous factors have been shown to govern clinical and laboratory efficacy standards, the role a clinician's or investigator's attitude plays in shaping the environment, and hence outcome measures is poorly understood. This study was designed to determine if subtle physical and/or verbal innuendo could act as a "soft" experimental bias, such that a physician or scientist could unwittingly bias outcome. This concept of "soft" bias is in contrast to the commonly known forms of "hard" experimental bias, such as the placebo or nocebo.

Commensurate with other first approximation studies this investigation leaves more questions unanswered such as:

1. Are there environmental factors (versus "hard" experimental bias) that may explain why laboratories on opposing sides of a specific question or procedure report findings that validate their own doctrine?

For example, Braud and Schlitz (9) performed a controlled, prospective double-blinded investigation on an electrodermal stress response. Wiseman and Schlitz (10) challenged the results, citing repeated protocol failures in his laboratory. He invited one of the authors, Marilyn Schlitz, to attempt to replicate her results in his laboratory.

Surprisingly, when Schlitz functioned as the chief investigator she successfully reproduced her original positive results - yet in an alternate trial when Wise- 
Table 1. Participant readings,

\begin{tabular}{|c|c|c|c|c|c|c|}
\hline \multicolumn{7}{|c|}{ Participant Readings (POSITIVE) } \\
\hline Variable & Reading 1 & Reading 2 & Reading 3 & Reading 4 & Total Readings & Average Reading \\
\hline 1 & 73 & 71 & 70 & 68 & 282 & 70.5 \\
\hline 2 & 79 & 76 & 75 & 74 & 304 & 76 \\
\hline 3 & 76 & 77 & 77 & 75 & 305 & 76.25 \\
\hline 4 & 55 & 59 & 61 & 57 & 232 & 58 \\
\hline 5 & 62 & 61 & 62 & 58 & 243 & 60.75 \\
\hline 6 & 64 & 60 & 61 & 58 & 243 & 60.75 \\
\hline 7 & 75 & 73 & 68 & 69 & 285 & 71.25 \\
\hline 8 & 89 & 80 & 79 & 80 & 328 & 82 \\
\hline 9 & 89 & 94 & 96 & 91 & 370 & 92.5 \\
\hline 10 & 96 & 97 & 94 & 98 & 385 & 96.25 \\
\hline \multicolumn{7}{|c|}{ Participant Readings (NEGATIVE) } \\
\hline Variable & Reading 1 & Reading 2 & Reading 3 & Reading 4 & Total Readings & Average Reading \\
\hline 1 & 68 & 73 & 70 & 66 & 277 & 69.25 \\
\hline 2 & 63 & 76 & 75 & 70 & 284 & 71 \\
\hline 3 & 83 & 71 & 71 & 71 & 296 & 74 \\
\hline 4 & 55 & 55 & 50 & 57 & 217 & 54.25 \\
\hline 5 & 63 & 60 & 58 & 56 & 237 & 59.25 \\
\hline 6 & 66 & 63 & 57 & 56 & 242 & 60.5 \\
\hline 7 & 65 & 60 & 64 & 60 & 249 & 62.25 \\
\hline 8 & 88 & 78 & 87 & 75 & 328 & 82 \\
\hline 9 & 88 & 89 & 90 & 89 & 356 & 89 \\
\hline 10 & 104 & 99 & 94 & 100 & 397 & 99.25 \\
\hline
\end{tabular}

man was the chief investigator, the experiment once again failed.

2. Does relationship centered healthcare affect outcome?

Within this context, some evidence suggests that the complex responsive processes of relating (CRPR) can be influenced by the reaction to others (11). For example a physician may be unable to shape a positive outcome to treatment of a patient he cannot relate to on a socioeconomic or cultural level. CRPR indicates that individuals must approach differences with curiosity rather than fear and defensiveness to effect a favorable relationship (11). This concept may call for a new perspective on the traditional "starched collar" physician/patient relationship paradigm that some have suggested breeds reticence to relinquish control. However, in order to understand the physician/patient
Table 2. T-test results.

\begin{tabular}{|c|c|c|c|c||}
\hline \multicolumn{5}{|c|}{ t-test } \\
\hline & n & Mean & SD & t \\
\hline Positive & 10 & 72.08 & 14.33 & $2.29^{\text {** }}$ \\
\hline Negative & 10 & 74.43 & 13.06 & $2.29^{\text {** }}$ \\
\hline
\end{tabular}

${ }^{* *} p \leq .05$

relationship better, there must first be a greater understanding of the environmental conditions affecting patient care outcome.

\section{Conclusions}

The study suggests that clinical environmental conditions influence the physical performance of a grip strength maneuver. Thus, it seems probable that clinical or experimental settings may affect diagnostic test results and/or functional outcome. 


\section{References}

1. Leonard K, Masatu M. Outpatient process quality evaluation and the Hawthorne effect. Soc Sci Med 2006; 63:2330-2340.

2. Street R, Krupat E, Bell R, Kravitz R, Haidet P. Beliefs about control in the physician-patient relationship. J Gen Intern Med 2003; 18:609-616.

3. Buller M, Buller D. Physicians' communication style and patient satisfaction. J Health Soc Behav 1987; 28:375-388.

4. Bertakis K, Helms L, Callahan E, Azari $R$, Robbins J. The influence of gender on physician practice style. Medical Care 1995; 33:407-416.
5. Hall J, Irish J, Roter D, Ehrlich C, Miller L. Satisfaction, gender, and communication in medical visits. Medical Care 1994; 32:1216-1231.

6. Basow S, Rubenfeld K. Troubles talk: Effects of gender and gender typing. Sex Roles 2003; 48:183-187.

7. Ashton A, Myers S. Serial grip strength testing - Its role in assessment of wrist and hand disability. The Internet Journal of Surgery 2004; 5:99-124.

9. Mathiowetz V. Reliability and validity of grip and pinch strength measurements. Critical Reviews in Physical and Rehabilitation Medicine 1991; 2:201-212.
9. Braud W, Schlitz. M. Psychokinetic in fluence on electrodermal activity. Journal of Parapsychology 1983; 47:95119.

10. Wiseman R, Schlitz M. Experimenter ef fects and the remote detection of starting: An attempted replication. The Journal of Parapsychology 1999; 61:471479.

11. Suchman A. A new theoretical foundation for relationship-centered care complex responsive processes of relating. J Gen Intern Med 2006; 21:40-44. 